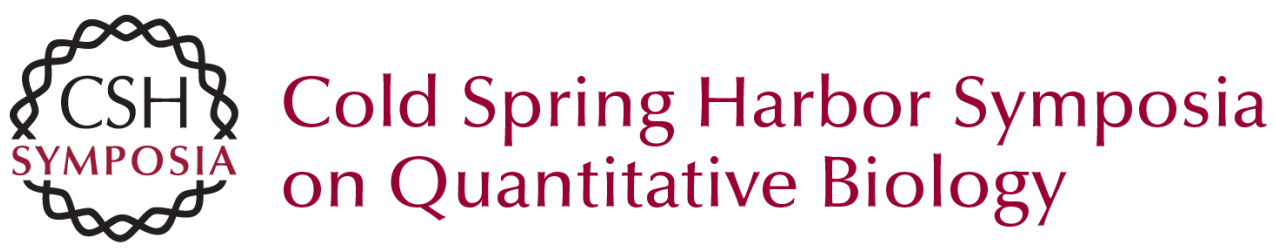

\title{
Protein Tyrosine Phosphatases: The Problems of a Growing Family
}

N.K. Tonks, Q. Yang, A.J. Flint, et al.

Cold Spring Harb Symp Quant Biol 1992 57: 87-94

Access the most recent version at doi:10.1101/SQB.1992.057.01.012

References This article cites 63 articles, 30 of which can be accessed free at: http://symposium.cshlp.org/content/57/87.refs.html

Article cited in:

http://symposium.cshlp.org/content/57/87\#related-urls

Email alerting

Receive free email alerts when new articles cite this article - sign up in service the box at the top right corner of the article or click here

To subscribe to Cold Spring Harbor Symposia on Quantitative Biology go to: http://symposium.cshlp.org/subscriptions 


\title{
Protein Tyrosine Phosphatases: The Problems of a Growing Family
}

\author{
N.K. Tonks, Q. YANG, A.J. Flint, M.F.B.G. Gebbink, B.R. FranzA, JR., * \\ D.E. HiLL, $\dagger$ H. Sun, and S. BRadY-KalnaY \\ Cold Spring Harbor Laboratory, Cold Spring Harbor, New York 11724; *Freeman Laboratory of Cancer Cell Biology, \\ Cold Spring Harbor Laboratory, Cold Spring Harbor, New York 11724; \\ $\uparrow$ Applied Biotechnology, Inc., Cambridge, Massachusetts 02138
}

Protein tyrosine phosphorylation is now recognized as an important component of the control of many fundamental aspects of cellular function, including growth and differentiation, cell cycle and cytoskeletal integrity. In vivo, the net level of phosphorylation of tyrosyl residues in a target substrate reflects the balance between the competing action of kinases and phosphatases. We are examining physiological roles for protein tyrosine phosphorylation, pursuing the problem from the perspective of the enzymes that catalyze the dephosphorylation reaction, the protein tyrosine phosphatases (PTPases). The PTPases have, until recently, been somewhat neglected relative to the protein tyrosine kinases (PTKs). However, considerable progress has been made in identifying new members of the PTPase family, and it appears that they constitute a novel class of signal transducing molecules that rival the PTKs in their structural diversity and complexity.

One of the principal reasons that the study of PTPases has lagged behind that of the kinases is a technical one-the requirement for a suitably purified phosphorylated protein substrate with which to assay phosphatase activity. In the case of tyrosine phosphorylation, which accounts for less than $0.1 \%$ of phosphate linked to protein (Hunter and Sefton 1980), the low abundance of modified proteins raises problems in obtaining sufficient quantities for their utilization in routine assays. To overcome this problem, a number of artificial substrates have been utilized, most notably a reduced carboxamidomethylated and maleylated (RCM) derivative of lysozyme. The major PTPase activity in human placenta extracts was purified to homogeneity (Tonks et al. 1988a,b) using RCM lysozyme as substrate and a thiophosphorylated derivative of the protein bound to Sepharose as an affinity support.

Determination of the amino acid sequence of the purified enzyme, termed PTP1B, illustrated two important points (Charbonneau et al. 1988). First, PTP1B was not structurally related to the serine/threonine phosphatases. Thus, whereas the protein kinases are derived from a common ancestor, the protein phosphatases appear to have evolved in separate families. Second, a striking level of sequence identity was detected between PTP1B and the tandem intracellular domains in the leukocyte common antigen, CD45. CD45 is an abundantly expressed, hematopoietic cell- surface protein that exists in multiple forms due to alternative mRNA splicing of 3 exons encoding sequences at its amino terminus (for review, see Thomas 1989). Its structure can be described in terms of a PTPase-related intracellular segment, a single transmembrane segment, and an extracellular domain that is highly glycosylated, is rich in cysteinyl residues, and possesses the hallmarks of a ligand-binding motif. This observation was exciting because it suggested that CD45 can be considered as a prototype of transmembrane, receptor-like PTPases with the potential to trigger signaling events at the cell membrane through the ligand-modulated dephosphorylation of tyrosyl residues in proteins. This concept is supported by the demonstration of intrinsic PTPase activity in CD45 (Tonks et al. 1988c).

These observations have set in motion a rapidly ex panding area of research involving many laboratories. At the present time, molecular biological approaches involving polymerase chain reaction (PCR) and lowstringency screening have identified 31 PTPases, excluding species homologs, as full-length sequences (for detailed review, see Charbonneau and Tonks 1992). Many additional partial sequences remain to be added to the list. The unique feature that defines the PTPase

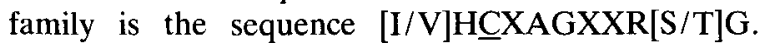
Within this motif, the cysteinyl residue is absolutely essential for activity, since the catalytic mechanism proceeds via the generation of a cysteine-phosphate intermediate (Guan and Dixon 1991). The structural features of several members of the PTPase family are illustrated in Figure 1.

\section{Receptor-linked PTPases}

With the exception of HPTP $\beta$ and a related molecule in Drosophila, DPTP10D, the transmembrane PTPases are characterized by the presence of two, tandemly repeated PTPase domains. The advantage of such an arrangement is unclear, although it should be remembered that oligomeric enzymes have the inherent capacity for cooperative interactions between their catalytic centers. However, it remains a point of controversy as to whether both domains are active (Streuli et al. 1990; Wang and Pallen 1991). For LAR and $\mathrm{CD} 45$, the idea has been proposed that domain 2 may 

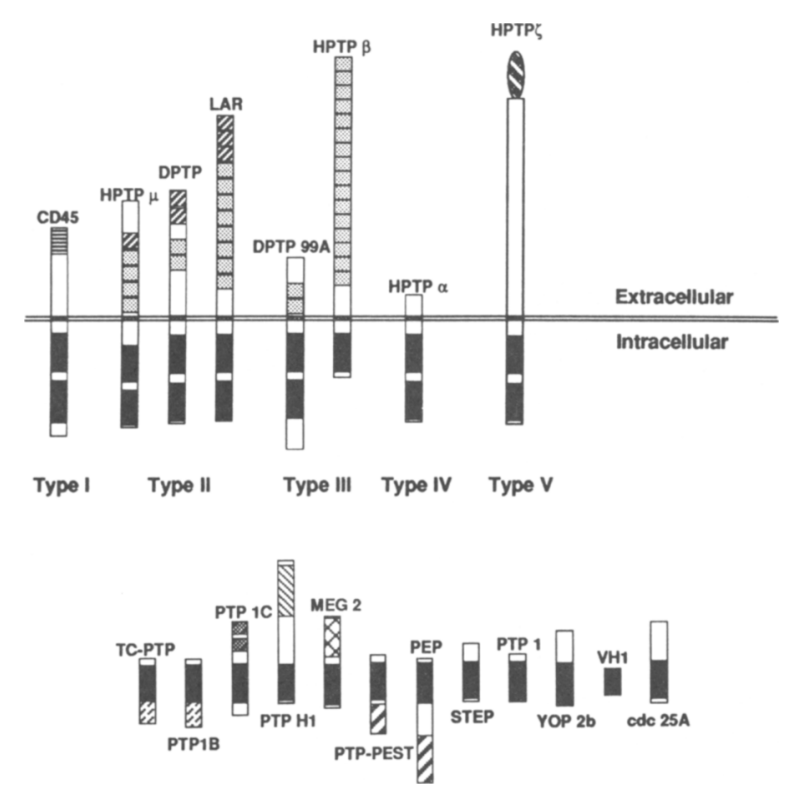

Figure 1. Members of the PTPase family. Conserved catalytic domains are shown in black. PTPs can be categorized as transmembrane, receptor-like, or nontransmembrane molecules. The receptor-like species can be subdivided into five types based on the structure of their extracellular segments. Type I represents the CD 45 family, multiple isoforms of which arise from differential splicing of a primary mRNA transcript of a single gene; three exons encoding sequences at the extreme amino terminus (horizontal lines) are differentially expressed. Type II contains Ig-like (diagonal lines) and tandem FN type III-like repeat domains (stippled); this category includes LAR, DLAR, DPTP, and HPTP $\mu$. Type III bears multiple FN type III-like repeats. Some type III isoforms such as HРTP $\beta$ have only one internal PTP domain. Type IV isoforms such as $\alpha$ and $\epsilon$ have small glycosylated extracellular segments. Type $\mathrm{V}$ possesses an amino-terminal motif with homology to carbonic anhydrase. Multiple nontransmembrane forms have also been identified. Many of the nonreceptor PTPs bear noncatalytic segments that are structurally related to other well-characterized proteins. The positions and relative sizes of these noncatalytic domains are shown as boxes containing distinct symbols; noncatalytic regions that have similar sequences are designated with identical patterns. The noncatalytic segments that have been identified include two SH2 domains in PTP1C, band 4.1 homology domains in PTPH1, an apparent lipid-binding domain in MEG2, and segments containing PEST sequences in PEP and PTP-PEST. In TC-PTP and PTP1B, the carboxy-terminal noncatalytic segments appear to play a role in modulating activity and controlling subcellular localization. PTP1, STEP, and Yop2b have noncatalytic sequences that are apparently unrelated to sequences in the databases. The protein from vaccinia virus, $\mathrm{VH} 1$, is much smaller than the other PTPs and presumably encodes only essential sequences within the catalytic domain. (Reprinted, with permission, from Tonks et al. 1993.)

itself not express activity but rather may function in modifying the specificity of domain 1 (Streuli et al. 1990). In support of this, inspection of the sequence of domain 2 in at least two PTPases, HPTP $\zeta$ and HPTP $\gamma$, illustrates that the catalytically essential cysteinyl residue is replaced by aspartate. In those enzymes in which the essential cysteine has been retained in domain 2 , there is the possibility that each domain may respond differently to potential ligands, display differ- ent substrate specificity, or be subjected to different modes of regulation. In the latter regard, it is interesting to note the unique 19-residue insert in domain 2 of CD45 that possesses potential sites of phosphorylation by casein kinase 2 (Charbonneau et al. 1988).

In contrast to the similarity among intracellular segments, the transmembrane PTPases can be readily distinguished by the structural diversity within their extracellular segments. At least five subtypes can be distinguished in this manner, as described in Figure 1. It should be stressed that these molecules must be considered putative receptors; no ligand has yet been identified which, upon binding to the extracellular segment of a transmembrane PTPase, alters the activity of the intracellular catalytic domains. The search for potential ligands remains a major challenge. A recent report suggested that CD45 may interact with a B-cell surface protein, $C D 22$, although the report did not address the consequences for CD45 activity of this binding interaction (Stamenkovic et al. 1991). That this interaction is specific for the $180-\mathrm{kD}$ form of CD45 also suggests that if in fact CD22 is a ligand for CD45, it is not the only one. As discussed below, the structural similarity between the type II receptor-like PTPases and adhesion molecules of the immunoglobulin superfamily suggests that these molecules may participate in homophilic binding interactions. In addition, the 280residue motif at the amino terminus of PTP $\zeta$ that is structurally related to carbonic anhydrase may form a binding pocket for low $M_{\mathrm{r}}$ ligands (Krueger and Saito 1992).

It is unclear what effect ligand binding will have on PTPase activity. Purified preparations of several receptor-like PTPases, including CD45 (Tonks et al. 1990), display considerable basal activity in vitro apparently in the absence of added ligand, raising the possibility that they are constitutively active in vivo. It is possible that ligand binding either inhibits activity or limits mobility of the enzyme on the membrane, thus restricting the spectrum of substrates with which it may interact.

\section{PTPases and Cell Adhesion}

The type II receptor-like PTPases are characterized by the presence of multiple immunoglobulin ( $\mathrm{Ig}$ ) and fibronectin (FN) type III-like repeats in the extracellular segment, which is characteristic of cell adhesion molecules such as N-CAMs (for review, see Edelman 1988). The first such PTPase identified was leukocyte common antigen-related (LAR) (Streuli et al. 1988), the 1234-residue extracellular segment of which contains 3 Ig-like and 8 FN III domains. LAR undergoes a posttranslational proteolytic cleavage to generate a mature protein that is a complex of two noncovalently associated subunits (Streuli et al. 1992; Yu et al. 1992). The $150-\mathrm{kD}$ E subunit is derived from the N-CAM-like extracellular segment, whereas the $85-\mathrm{kD}$ P subunit comprises the intracellular and transmembrane segments as well as a short stretch of extracellular se- 
quence that interacts with the E subunit. Interestingly, in HeLa cells, the E subunit is shed in a cell-densitydependent manner; however, the effects of dissociation of $E$ and $P$ subunits on activity remain to be ascertained. In Drosophila, a role for DLAR and the other type II enzymes DPTP10D and DPTP99A (Hariharan et al. 1991; Tian et al. 1991; Yang et al. 1991) in cell adhesion and development has been suggested. In situ hybridization and immunocytochemistry data indicate that expression of these PTPases is restricted to the central nervous system, where the timing and pattern of their appearance on axons is consistent with a role in regulating neurite outgrowth and pathfinding during development.

We are particularly interested in a novel, receptorlike PTPase, termed RPTP $\mu$, the sequence of which was reported by Gebbink et al. (1991). The cDNA predicts a protein of $\sim 162 \mathrm{kD}$ in which the extracellular segment includes one Ig-like and four FN III-like domains. There is a single transmembrane domain, and the intracellular segment bears two PTPase domains. This molecule is also characterized by the presence of a 158-residue juxtamembrane sequence that is some 70 residues longer than the equivalent segment in other receptor PTPases. We have also isolated cDNA for the human homolog of this molecule from a HeLa cell library (Q. Yang and N.K. Tonks, unpubl.). We have noted an intriguing similarity between the sequence of the juxtamembrane segment of this PTPase and that of the intracellular segment of the cadherin family of cell adhesion molecules (Fig. 2).

Cadherins are adhesion molecules that participate in the mechanisms of coordinated association of cells during tissue development and morphogenesis (for review, see Magee and Buxton 1991; Takeichi 1991). Essentially all cells that associate into tissues express cadherins, and reduction in the level of expression of E-cadherin has been linked with tumor invasiveness and metastasis (Frixen et al. 1991). Cadherins are concentrated in adherens-type junctions, where they are linked to cortical actin bundles. Four subclasses of cadherins have been well characterized at a structural level: L-CAM (liver cell adhesion molecule) in chickens, and E(epithelial)-, N(neural)-, and $\mathbf{P}$ (placental)-cadherins in mammals. However, the repertoire of cadherins is also now expanding rapidly (Napolitano et al. 1991; Suzuki et al. 1991). Their structure can be described in terms of three segments. The extracellular segment is

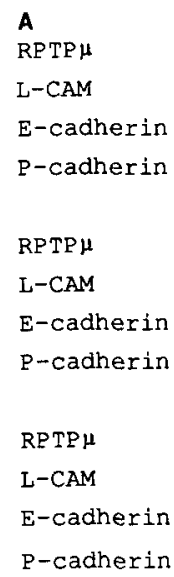

B-cadherin

RPTPH
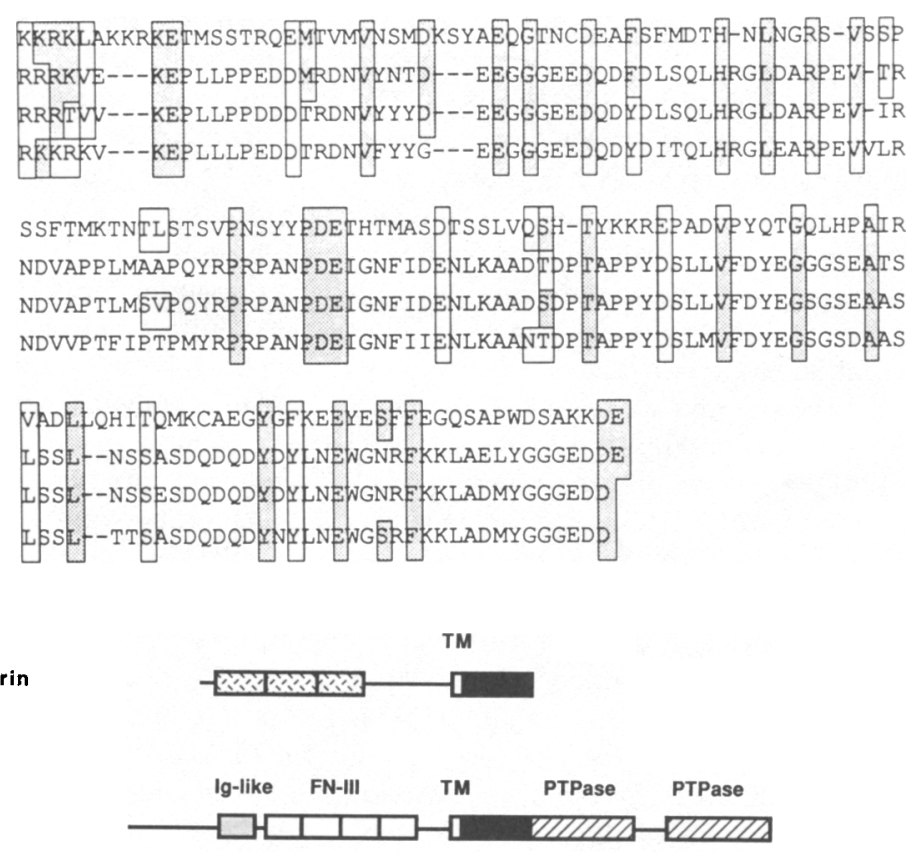

PTPase 1B

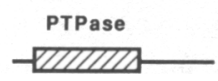

Figure 2. ( $A$ ) Alignment of amino acid residues in the juxtamembrane domain of PTP $\mu$ with the conserved cytoplasmic domains of cadherins. Identities are highlighted in shaded boxes and conservative substitutions in open boxes. $(B)$ Diagram illustrating the structure of PTP $\mu$ as well as its relationship to the cadherins and the low $M_{\mathrm{r}}$ PTPase, PTP1B. Diagonal lines denote PTPase catalytic domains. The segment of homology between PTP $\mu$ and the cadherins is represented in black. The transmembrane segments also share 8 identities. Using the ALIGN program, the mutation data matrix for scoring, and a gap penalty of 10 to compare residues 742-917 in PTP $\mu$ (Gebbink et al. 1991) with 553-722 in L-CAM (Gallin et al. 1987) and 555-724 in E-cadherin (Ringwald et al. 1987), scores of 7.3 and 7.5, respectively, were obtained. These alignment scores are expressed in units of standard deviation from the average background scores of 100 randomly generated sequences. Scores greater than 5 indicate homology. 
glycosylated and comprises at least three repeated structural motifs containing stretches of acidic residues involved in binding $\mathrm{Ca}^{++}$. Cadherin binding is homophilic and $\mathrm{Ca}^{++}$-dependent; binding specificity is governed by the amino-terminal 113 residues of the extracellular segment. There is a single transmembrane domain and an intracellular segment of $\sim 150$ residues. It is the intracellular segment that is most highly conserved, displaying $\sim 90 \%$ identity among the cadherins. Extensive analysis by site-directed mutagenesis has shown that deletions in the intracellular segment of $\mathrm{E}$ - and $\mathrm{N}$-cadherin, particularly the carboxy-terminal 72 amino acids, abolish adhesion despite the presence of an intact extracellular segment (Nagafuchi and Takeichi 1988). The intracellular segment interacts with cortical actin indirectly, a process that is mediated by the binding of cytoplasmic proteins termed catenins. Catenin $\alpha(102 \mathrm{kD})$ displays homology with vinculin (Nagafuchi et al. 1991), catenin $\beta$ (92-94 kD) displays homology with plakoglobin (McCrea et al. 1991), and catenin $\gamma(\sim 80 \mathrm{kD})$ remains to be characterized. Deletions of the carboxy-terminal sequences of cadherin also abolish the binding to catenins. Thus, the association of the cadherin cytoplasmic domain, the domain that displays homology with $\mathrm{PTP} \mu$, with catenins is essential for the interaction of cadherins with the cytoskeleton and their adhesive function.

What is the meaning of this homology between PTP $\mu$ and cadherins? We propose that this PTPase is directed to cytoskeletal targets through interaction between the juxtamembrane domain and molecules functionally similar to the catenins, thus localizing it to points of interface between the plasma membrane and the cytoskeleton. Interestingly, the adherens junctions have recently been identified as major sites of tyrosine phosphorylation within the cell and sites at which members of the src family of tyrosine kinases are concentrated (Tsukita et al. 1991). Furthermore, treatment of chick lens cells with inhibitors of PTPase activity leads to a pronounced accumulation of phosphotyrosine at adherens junctions, implying a ready reversibility of the phosphorylation reaction and a rapid turnover of tyrosine phosphate at these sites (Volberg et al. 1991).

The consensus view appears to be that although interaction with the cytoskeleton is crucial for the cell adhesion function of cadherins, this is not the case for members of the Ig-superfamily of adhesion molecules, such as N-CAM. In fact, the smallest form of N-CAM, termed ssd, lacks transmembrane and cytoplasmic segments and is attached to the external face of the membrane by a phosphatidylinositol glycan anchor (Edelman 1988). Nevertheless, there are reports of the triggering of signal transduction pathways involving Gprotein-mediated changes in the levels of second messengers such as $\mathrm{Ca}^{++}$by N-CAM and N-cadherin (Schuch et al. 1989; Doherty et al. 1991). РTP $\mu$ may provide a link between cell adhesion and signal transduction pathways involving reversible tyrosine phosphorylation. We also suggest that the cadherin-related juxtamembrane domain of $\mathrm{PTP} \mu$ may regulate the interaction of this Ig-superfamily molecule with the cytoskeleton in addition to controlling its potential adhesive function in a manner analogous to the cadherins. The identification of the proteins with which РTP $\mu$ interacts should provide new insights into the role of reversible tyrosine phosphorylation in control of the cytoskeleton and cell adhesion.

\section{Nontransmembrane PTPases}

Generally, the PTPases are multidomain proteins bearing at least one catalytic domain and additional, noncatalytic, distinct structural motifs that appear to be important in regulation, including targeting to particular subcellular locations. Whereas the receptor-like PTPases have the potential for modulation of activity by binding of a ligand to the extracellular segment, several nontransmembrane PTPases, including two recently identified in our laboratory, further illustrate this general principle.

The structure of PTPH1 can be described in terms of three segments (Yang and Tonks 1991): an aminoterminal domain of $\sim 320$ residues that displays homology with the similarly located domains in band 4.1, ezrin, and talin; a central segment bearing several putative sites for phosphorylation by $\mathrm{p} 34^{\mathrm{cdc} 2}$ and casein kinase 2; and a carboxy-terminal catalytic domain. Intrinsic activity in PTPH1 has now been demonstrated (N.K. Tonks et al., unpubl.). In erythrocytes, band 4.1 promotes the association of actin and spectrin and, through its amino-terminal domain, binds to the transmembrane protein glycophorin in a phosphatidylinositol bisphosphate-dependent manner. Similar interactions between talin and $\beta$-integrin at focal adhesion plaques have also been proposed. Thus, it appears that this amino-terminal domain defines a family of proteins that are targeted to the interface between the plasma membrane and the cytoskeleton, suggesting that PTPH1 will display a similar subcellular location. Complementary DNA for a similar, but distinct, PTPase termed MEG01 has been isolated by Gu et al. (1991).

PTP-PEST is a widely expressed PTPase comprising an amino-terminal catalytic domain, in which intrinsic PTPase activity has also been demonstrated, and a carboxy-terminal segment that is rich in proline, glutamic acid, serine, and threonine residues and is characterized by the presence of PEST sequences (Yang et al. 1993). PEST sequences are a characteristic of proteins with short intracellular half-lives (for review, see Dice 1987). Such potential susceptibility to rapid degradation could be thought of as "terminal" targeting or targeting to destruction. Interestingly, like many PEST-sequence-containing proteins, PTP-PEST is the product of an inducible gene. In human rhabdomyosarcoma A204 muscle cells, stimulation with insulin leads to an increase of approximately fourfold in PTP-PEST mRNA levels. Possible links between PTPPEST and signal transduction through the insulin re- 
ceptor are under investigation. Another PESTsequence-containing PTPase, PEP, was isolated recently by Matthews et al. (1992). It is a distinct gene product $\sim 200$ residues longer than PTP-PEST.

Recently, it has become apparent that there exists a new subfamily of PTPases which possess the conserved sequence motif surrounding the essential cysteinyl residue ([I/V]HCXAGXXR[S/T]G) but display little structural similarity elsewhere in the molecule to the other members of the family (Fig. 3). The founder member of this subfamily is the VH1 protein from the poxvirus vaccinia (Guan et al. 1991). Unlike the PTPases described thus far, which are absolutely specific for tyrosyl residues in proteins, $\mathrm{VH} 1$ displays dual specificity, dephosphorylating both phosphotyrosyl and phosphoseryl residues in vitro. Additional members now include the growth-factor-inducible murine immediate-early gene product $3 \mathrm{CH} 134$ (Charles et al. 1992) and its human homolog, CL100 (Keyse and Emslie 1992); the cell cycle control element p80 ${ }^{\text {cdc25 }}$, which is the phosphatase that dephosphorylates and activates $\mathrm{p} 34^{\mathrm{cdc} 2}$ at the $\mathrm{G}_{2} / \mathrm{M}$ phase transition of the eukaryotic cell cycle (Strausfeld et al. 1991); CDC14 from Saccharomyces cerevisiae (Wan et al. 1992); and an ORF from Autographa californica nuclear polyhedrosis virus (Tilakaratne et al. 1991).

Although it was believed that the exclusive provenance of tyrosine phosphorylation was eukaryotic cells, several reports have indicated the presence of phosphotyrosine in prokaryotes (Dadssi and Cozzone 1990; Atkinson et al. 1992). In addition, Dixon's group has ascertained that the essential virulence determinant of the bacterium Yersinia, the causative agent of the plague or Black Death, is a PTPase termed Yop2b, the activity of which is essential for virulence (Guan and Dixon 1990; Bliska et al. 1991). The Yop2b protein is encoded by the YopH gene located on a $70-\mathrm{kb}$ megaplasmid. Rather than endogenous substrates, its targets are tyrosine phosphorylated proteins in the infected eukaryotic host cells. Thus, it has been suggested that the YopH gene may have been acquired through lateral gene transfer from a eukaryotic host, as suggested for retroviral PTKs. However, more recently, in the cyanobacterium Nostoc commune, a chromosomally encoded ORF termed IphP has been identified which, like VH1, displays sequence identity with the PTPases essentially only in the segment surrounding the active-site cysteine. Not only does the IphP gene encode an active phosphatase which, like VH1, displays dual specificity,

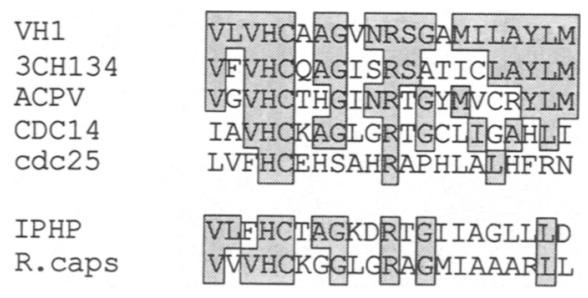

Figure 3. The VH1-related subfamily of PTPases. but also a number of potential endogenous substrates have been identified (M. Potts et al., in prep.). We have also observed a PTPase-related ORF in the bacterium, Rhodobacter capsulatus (Protein Identification Resource Database, No. S19740). Such observations suggest that tyrosine phosphorylation may have arisen as a regulatory modification of proteins much earlier in evolutionary time than previously anticipated.

\section{Regulation of PTPase Activity}

Initial measurements of the activity of PTP1B and CD45 indicated a very high $V_{\max }, 10$ - to 1000 -fold higher than that of the PTKs, and a high affinity for substrate in vitro (Tonks et al. 1988b, 1990). Thus, the PTPases have the potential to represent formidable barriers to the action of PTKs in vivo, suggesting that mechanisms must exist to harness their activity. We are studying such control mechanisms for several of the PTPases. As discussed above, many PTPases possess structural motifs suggestive of regulation through intracellular targeting. Superimposed on control mechanisms mediated through structural features of the enzyme is the potential for regulation by phosphorylation of the phosphatase.

There have been preliminary reports of phosphorylation of PTPases in vivo. For example, CD45 becomes phosphorylated on tyrosyl residues following stimulation of Jurkat $\mathrm{T}$ cells with phytohemagglutinin or anti-CD3 antibodies (Stover et al. 1991). Changes in phosphoserine levels in CD45 have also been reported; it is phosphorylated in response to phorbol ester stimulation of peripheral $\mathrm{T}$ cells (Autero and Gahmberg 1987), whereas stimulation of various $\mathrm{T}$-cell lines with ionomycin, to mobilize intracellular $\mathrm{Ca}^{++}$, leads to its dephosphorylation (Ostergaard and Trowbridge 1991). Our studies in this direction currently focus on PTP1B. Although it was originally purified as a monomeric catalytic subunit of $37 \mathrm{kD}$, isolation of cDNA for PTP1B predicted a longer protein of 435 amino acids $(\sim 50 \mathrm{kD})$ with an extension of 114 amino acids at the carboxyl terminus. This carboxy-terminal segment serves a regulatory function. The extreme carboxyterminal 35 residues are both necessary and sufficient for targeting to membranes of the endoplasmic reticulum (ER) (Frangioni et al. 1992). The preceding 88 amino acids are predominantly hydrophilic and bear several putative sites for phosphorylation by serine/ threonine kinases. We have demonstrated that PTP1B from HeLa cells is phosphorylated in vivo (Fig. 4) on seryl residues. In view of the fact that activation of the serine/threonine kinase $p 34^{\text {cdc2 }}$ occurs at the $G_{2} / M$ phase transition of the cell cycle (for review, see Murray 1992 ), the possibility that $\mathrm{p} 34^{\text {cdc2 }}$ was responsible for this phosphorylation was considered. Indeed, there are at least two sites in PTP1B that are phosphorylated in mitosis, of which one, S386, is a substrate for $\mathrm{p} 34^{\mathrm{cdc} 2}$ both in vitro and in vivo. There is a second site, S352, phosphorylation of which accounts for the pronounced retardation in electrophoretic mobility of PTP1B de- 


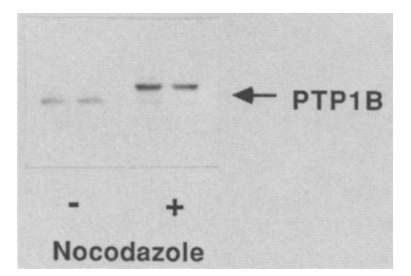

Figure 4. PTP1B is phosphorylated in a cell-cycle-dependent manner in HeLa cells. PTP1B was immunoprecipitated from ${ }^{32}$ P-labeled HeLa cells using a monoclonal antibody FG6 and subjected to SDS polyacrylamide gel electrophoresis. The figure highlights the section of the gel containing PTP1B, identified by autoradiography.

tected in mitotic cells (Fig. 4). This site is not phosphorylated by $\mathrm{p} 34^{\mathrm{cdc} 2}$ in vitro or in vivo, and the identity of the kinase involved remains to be established. The precise role of this mitotic phosphorylation is also unclear. There appears to be no gross change in intracellular localization of PTP1B coupled with the transition from $G_{2}$ to $M$ phase, since the enzyme remains in a membrane fraction. However, a more detailed immunocytochemical examination is yet to be completed. When the activity of PTP1B from mitotic cells is measured in vitro with artificial substrates, a small inhibition is detected relative to the activity of this enzyme in asynchronous populations. It is possible that a more profound effect will be observed with a physiologically relevant substrate. The identity of the physiological substrates for PTP1B is unknown. Its localization raises the possibility of a role in controlling the dynamic changes in ER structure and association with the cytoskeleton in interphase cells (Lee and Chen 1988) or in the assembly/disassembly of this subcellular compartment with the cell cycle. The demonstration of ER localization of Ltk (Bauskin et al. 1991) also suggests that this tyrosine kinase or its substrates may be susceptible to the action of PTP1B in vivo.

In addition to mitotic phosphorylation, PTP1B is phosphorylated on S378 in vitro by PKC and on the same site in HeLa cells in vivo in response to TPA stimulation. There was no discernible effect of this modification on activity in vitro. Thus, PTP1B represents a point of convergence for the action of at least three serine/threonine kinases, suggestive of an important role in signal transduction in vivo. Such phosphorylation of a PTPase may be indicative of a new tier of control of the level of cellular phosphotyrosine and a novel mode of interaction between steps in signaling pathways involving serine/threonine and tyrosine phosphorylation.

\section{PERSPECTIVES}

Although research into the PTPases is largely in an exploratory phase where progress is primarily being made in the identification of new isoforms, the predicted structures of these enzymes are clearly consistent with important and diverse roles in controlling signal transduction in vivo. Several lines of evidence now dispel the notion that the PTPases are merely constitutively active housekeeping enzymes that function solely to antagonize the action of PTKs. First, the generation of various $\mathrm{CD}_{4} 5^{-}$cell lines indicates that $\mathrm{CD} 45$ is essential for antigen-induced T-cell proliferation responses, for coupling of the T-cell receptor to the generation of phosphatidylinositol second messengers, and for the rapid increases in protein tyrosine phosphorylation associated with triggering of the T-cell receptor (for review, see Trowbridge 1991). It is suggested that these effects are mediated by the dephosphorylation and activation of members of the $s r c$ family of PTKs by CD45. Similarly, CD 45 has also been implicated in signaling through the B-cell receptor. Second, a key element in the control of the eukaryotic cell cycle is the dephosphorylation and activation of $\mathrm{p} 34^{\mathrm{cdc} 2}$ by cdc25 (for review, see Murray 1992). Third, it has recently been shown that in rat embryo fibroblasts overexpressing the transmembrane enzyme RPTP $\alpha$, there is a persistent activation of c-src with concomitant cellular transformation (Zheng et al. 1992). Finally, the Drosophila gene corkscrew, which encodes an SH2domain-containing PTPase, functions in concert with D-raf to transduce positively the signal from torso, a receptor PTK, in embryonic cell fate determination (Perkins et al. 1992). Such studies suggest that PTPases have the potential to serve as an "on-switch," acting positively in triggering signal transduction events.

Evidence is accumulating in several laboratories, including our own (McGuire et al. 1991), to implicate a dysfunction in the action of PTPases in type II diabetes. Study of the PTPases may also yield important information regarding various neoplasias. It has already been demonstrated that overexpression of PTP1B can confer on 3T3 cells resistance to transformation by oncogenic neu (Brown-Shimer et al. 1992) and can at least partially antagonize the action of v-src (Woodford-Thomas et al. 1992). In addition, there are suggestions that some PTPases may be the products of tumor suppressor genes (LaForgia et al. 1991), i.e., deletion of a PTPase may contribute to the elevation of phosphotyrosine in some transformed cells. One would anticipate that further study of the structure, function, and mode of regulation of members of the PTPase family will not only furnish us with a more complete understanding of the physiological significance of tyrosine phosphorylation, but may also offer targets for pharmacological intervention in several human diseases.

\section{ACKNOWLEDGMENTS}

N.K.T. is a Pew Scholar in the Biomedical Sciences and acknowledges support from the National Cancer Institute (CA-53840), ICOS Corporation, and the Hansen Foundation. B.R.F.'s laboratory is supported by a grant (CA-40512) from the National Cancer Institute. A.J.F. is supported by a Cancer Research Institute/F.M. Kirby Foundation fellowship, M.F.B.G.G. by the Dutch Cancer Society, and H.S. by a Damon Runyon-Walter Winchell Cancer Research Fund fel- 
lowship. We thank Carol Marcincuk for typing the manuscript.

\section{REFERENCES}

Atkinson, M., C. Allen, and L. Sequeira. 1992. Tyrosine phosphorylation of a membrane protein from Pseudomonas solanacearum. J. Bacteriol. 174: 4356.

Autero, M. and C.G. Gahmberg. 1987. Phorbol diesters increase the phosphorylation of the leukocyte common antigen CD45 in human T cells. Eur. J. Immunol. 17: 1503.

Bauskin, A.R., I. Alkalay, and Y. Ben-Neriah. 1991. Redox regulation of a protein tyrosine kinase in the endoplasmic reticulum. Cell 66: 685 .

Bliska, J.B., K. Guan, J.E. Dixon, and S. Falkow. 1991. Tyrosine phosphate hydrolysis of host proteins by an essential Yersinia virulence determinant. Proc. Natl. Acad. Sci. 88: 1187.

Brown-Shimer, S., K.A. Johnson., D.E. Hill, and A.M. Bruskin. 1992. Effect of protein tyrosine phosphatase 1B expression on transformation by the human neu oncogene. Cancer Res. 52: 478 .

Charbonneau, H. and N.K. Tonks. 1992.1002 protein phosphatases? Annu. Rev. Cell Biol. 8: 463.

Charbonneau, H., N.K. Tonks, K.A. Walsh, and E.H. Fischer. 1988. The leukocyte common antigen (CD45): A putative receptor-linked protein tyrosine phosphatase. Proc. Natl. Acad. Sci. 85: 7182.

Charles, C.H., A.S. Abler, and L.F. Lau. 1992. cDNA sequence of a growth factor-inducible immediate early gene and characterization of its encoded protein. Oncogene 7: 187 .

Dadssi, M. and A.J. Cozzone. 1990. Evidence of protein tyrosine kinase activity in the bacterium Acinetobacter cal. coaceticus. J. Biol. Chem. 265: 20996.

Dice, J.F. 1987. Molecular determinants of protein half lives in eukaryotic cells, FASEB J. 1:349.

Doherty, P., S.V. Ashton, S.E. Moore, and F.S. Walsh. 1991. Morpho-regulatory activities of NCAM and N-cadherin can be accounted for by $\mathrm{G}$ protein-dependent activation of L- and N-neuronal calcium channels. Cell 67: 21 .

Edelman, G.M. 1988. Morphoregulatory molecules. Biochemistry 27: 3533 .

Frangioni, J.V., P.H. Beahm, V. Shifrin, C.A. Jost, and B.G. Neel. 1992. The nontransmembrane tyrosine phosphatase PTP1B localizes to the endoplasmic reticulum via its 35 amino acid C-terminal sequence. Cell 68: 545 .

Frixen, U.H., J. Behrens, M. Sachs, G.. Eberle, B. Voss, A. Warda, D. Lochner, and W. Birchmeier. 1991. E-cadherinmediated cell-cell adhesion prevents invasiveness of human carcinoma cells. J. Cell Biol. 113: 173.

Gallin, W.J., B.C. Sorkin, G.M. Edelman, and B.A. Cunningham. 1987. Sequence analysis of a cDNA clone encoding the liver cell adhesion molecule, L-CAM. Proc. Natl. Acad. Sci. 84: 2808.

Gebbink, M.F.B.G., I. Van Etten, G. Hateboer, R. Suijkerbuijk, R.L. Beijersbergen, A.G. Van Kessel, and W.H. Moolenaar. 1991. Cloning, expression and chromosomal localization of a new putative receptor-like protein tyrosine phosphatase. FEBS Lett. 290: 123.

Gu, M.J., D. York, 1. Warshawsky, and P.W. Majerus. 1991. Identification, cloning and expression of a cytosolic megakaryocyte protein tyrosine phosphatase with sequence homology to cytoskeletal protein 4.1. Proc. Natl. Acad. Sci. 88: 5867.

Guan, K. and J.E. Dixon. 1990. Protein tyrosine phosphatase activity of an essential virulence determinant in Yersinia. Science 249: 5553

- 1991. Evidence for protein-tyrosine-phosphatase catalysis proceeding via a cysteine-phosphate intermediate. J. Biol. Chem. 266: 17026.

Guan, K., S.S. Broyles, and J.E. Dixon. 1991. A Thr/Ser protein phosphatase encoded by vaccinia virus. Nature 350: 359 .

Hariharan, I.K., P.T. Chuang, and G.M. Rubin. 1991. Cloning and characterization of a receptor-class phosphotyrosine phosphatase gene expressed on central nervous system axons in Drosophila melanogaster. Proc. Natl. Acad. Sci. 88: 11266 .

Hunter, T. and B.M. Sefton. 1980. The transforming gene product of Rous sarcoma virus phosphorylates tyrosine. Proc. Natl. Acad. Sci. 77: 1311.

Keyse, S.M. and E.A. Emslie. 1992. Oxidative stress and heat shock induce a human gene encoding a protein tyrosine phosphatase. Nature 359: 644 .

Krueger, N.X. and H. Saito. 1992. A human transmembrane protein tyrosine phosphatase, PTP $\zeta$, is expressed in brain and has an $\mathrm{N}$ terminal receptor domain homologous to carbonic anhydrases. Proc. Natl. Acad. Sci. 89: 7417.

LaForgia, S., B. Morse, J. Levy, G. Barnea, L.A. Cannizzaro, F. Li., P.C. Nowell, L. Boghosian-Sell, J. Glick, A. Weston, C.C. Harris, H. Drabkin, D. Patterson, C.M. Croce, J. Schlessinger, and K. Huebner. 1991. Receptor protein tyrosine phosphatase $\gamma$ is a candidate tumor suppressor gene at human chromosome region $3 \mathrm{p} 21$. Proc. Natl. Acad. Sci. 88: 5036.

Lee, C. and L.B. Chen. 1988. Dynamic behavior of endoplasmic reticulum in living cells. Cell 54: 37 .

Magee, A.I. and R.S. Buxton. 1991. Transmembrane molecular assemblies regulated by the greater cadherin family. Curr. Opin. Cell Biol. 3: 854.

Matthews, R.J., D.B. Bowne, E. Flores, and M.L. Thomas, 1992. Characterization of haematopoietic intracellular protein tyrosine phosphatases: Description of a phosphatase containing an SH2 domain and another enriched in proline, glutamic acid, serine and threonine rich sequences. Mol. Cell. Biol. 12: 2396.

McCrea, P., C.W. Turck, and B. Gumbiner. 1991. A homolog of the Armadillo protein in Drosophilia "Plakoglobin" associated with E cadherin. Science 254: 1359.

McGuire, M.C., R.M. Fields, B.L. Nyomba, I. Raz, C. Bogardus, N.K. Tonks, and J. Sommercorn. 1991. Abnormal regulation of protein tyrosine phosphatase activities in skeletal muscle of insulin-resistant humans. Diabetes 40: 939 .

Murray, A.W. 1992. Creative blocks: Cell cycle check points and feedback controls. Nature 359: 599.

Nagafuchi, A. and M. Takeichi. 1988. Cell binding function of E-cadherin is regulated by the cytoplasmic domain. EMBO J. 7: 3679 .

Nagafuchi, A., M. Takeichi, and S. Tsukita. 1991. The $102 \mathrm{kD}$ cadherin-associated protein: Similarity to vinculin and post transcriptional regulation of expression. Cell 65: 849 .

Napolitano, E.W., K. Venstrom, E.F. Wheeler, and L.F. Reichardt. 1991. Molecular cloning and characterization of B-cadherin, a novel chick cadherin. J. Cell Biol. 113: 893.

Ostergaard, H.L. and I.S. Trowbridge. 1991. Negative regulation of CD45 protein tyrosine phosphatase activity by ionomycin in T cells. Science 253: 1423.

Perkins, L.A., I. Larsen, and N. Perrimon. 1992. corkscrew encodes a putative protein tyrosine phosphatase that functions to transduce the terminal signal from the receptor tyrosine kinase torso. Cell 70: 225.

Ringwald, M., R. Schuh, D. Vestweber, H. Eiskelter, F. Lottspeich, J. Engel, R. Dolz, F. Jahnig, J. Epplen, S. Mayer, C. Muller, and R. Kemler. 1987. The structure of cell adhesion molecule uvomorulin. Insights into the molecular mechanism of $\mathrm{Ca}^{2+}$-dependent cell adhesion. EMBO J. 6: 3647

Schuch, U., M.J. Lohse, and M. Schachner. 1989. Neural cell adhesion molecules influence second messenger systems. Neuron 3: 13.

Stamenkovic, I., D. Sgroi, A. Aruffo, M.S. Sy, and T. Anderson. 1991. The B lymphocyte adhesion molecule CD22 interacts with leukocyte common antigen CD45RO on $\mathrm{T}$ 
cells and $\alpha 2-6$ sialyltranferase, CD75, on B cells. Cell 66: 1133 .

Stover, D.R., H. Charbonneau, N.K. Tonks, and K.A. Walsh. 1991. Protein tyrosine phosphatase CD45 is phosphorylated transiently on tyrosine upon activation of Jurkat T cells. Proc. Natl. Acad. Sci. 88: 7704.

Strausfeld, U., J.C. Labbé, D. Fesquet, J.C. Cavadore, A. Pacard, K. Sadhu, P. Russell, and M. Dorée. 1991. Dephosphorylation and activation of a $\mathrm{p} 34^{\mathrm{cdc2}} /$ cyclin B complex in vitro by human CDC25 protein. Nature 351: 242.

Streuli, M., N.X. Krueger, L.R. Hall, S.F. Schlossman, and H. Saito. 1988. A new member of the immunoglobulin superfamily that has a cytoplasmic region homologous to the leukocyte common antigen. J. Exp. Med. 168: 1523.

Streuli, M., N.X. Krueger, T. Thai, M. Tang, and H. Saito. 1990. Distinct functional roles of the two intracellular phosphatase-like domains of the receptor-linked protein tyrosine phosphatases LCA and LAR. EMBO J. 9: 2399.

Streuli, M., N.X. Krueger, P.D. Ariniello, M. Tang, J.M. Munro, W.A. Blattler, D.A. Adler, C.M. Disteche, and H. Saito, 1992. Expression of the receptor-linked protein tyrosine phosphatase LAR: Proteolytic cleavage and shedding of the CAM-like extracellular region. EMBO J. 11: 897 .

Suzuki, S., K. Sano, and H. Tanihara. 1991. Diversity of the cadherin family: Evidence for 8 new cadherins in nervous tissue. Cell Regul. 2: 261.

Takeichi, M. 1991. Cadherin cell adhesion receptors as a morphogenetic regulator. Science 251: 1451.

Thomas, M.L. 1989. The leukocyte common antigen family. Annu. Rev. Immunol. 7: 339.

Tian, S.S., P. Tsoulfas, and K. Zinn. 1991. Three receptorlinked protein phosphatases are selectively expressed on central nervous system axons in the Drosophila embryo. Cell 67: 675 .

Tilakaratne, N., S.E. Hardin, and R.F. Weaver. 1991. Nucleotide sequence and transcript mapping of the HindIIIF region of the Autographa californica nuclear polyhedrosis virus genome. J. Gen. Virol. 72: 285.

Tonks, N.K., C.D. Diltz, and E.H. Fischer. 1988a. Purification of the major protein tyrosine phosphatases of human placenta. J. Biol. Chem. 263: 6722.

. 1988b. Characterization of the major protein tyrosine phosphatases of human placenta. J. Biol. Chem. 263: 6731 .

- 1990. CD45, an integral membrane protein tyrosine phosphatase. J. Biol. Chem. 265: 10674.

Tonks, N.K., H. Charbonneau, C.D. Diltz, E.H. Fischer, and K.A. Walsh. 1988c. Demonstration that the leukocyte common antigen $\mathrm{CD} 45$ is a protein tyrosine phosphatase. Biochemistry 27: 8695 .

Tonks, N.K., A.J. Flint, M.F.B.G. Gebbink, H. Sun, and Q. Yang. 1993. Signal transduction and protein tyrosine dephosphorylation. Adv. Messenger Phosphoprotein Res. 28: (in press).

Trowbridge, I.S. 1991. CD45: A prototype for transmembrane protein tyrosine phosphatases. J. Biol. Chem. 266: 23517.

Tsukita, S., K. Oishi, T. Akiyama, Y. Yamanashi, T. Yamamoto, and S. Tsukita. 1991. Specific proto-oncogenic tyrosine kinases of $s r c$ family are enriched in cell-to-cell adherens junctions where the level of tyrosine phosphorylation is elevated. J. Cell Biol. 113: 867 .

Volberg, T.. B. Geiger, R. Dror, and Y. Zick. 1991. Modulation of intercellular adherens-type junctions and tyrosine phosphorylation of their components in RSV-transformed cultured chicken cells. Cell Regul. 2: 105.

Wan, J., H. Xu, and M. Grunstein. 1992. CDC14 of Saccharomyces cerevisiae. J. Biol. Chem. 267: 11274.

Wang, Y. and C.J. Pallen. 1991. The receptor-like protein tyrosine phosphatase HPTP $\alpha$ has two active catalytic domains with distinct substrates specificities. EMBO J. 10: 3231 .

Woodford-Thomas, T.A., J.D. Rhodes, and J.E. Dixon. 1992. Expression of a protein tyrosine phosphatase in normal and v-src-transformed mouse 3T3 fibroblasts. J. Cell Biol. 117: 401

Yang, Q. and N.K. Tonks. 1991. Isolation of a cDNA clone encoding a human protein tyrosine phosphatase with homology to the cytoskeletal-associated proteins band 4.1, ezrin and talin. Proc. Natl. Acad. Sci. 88: 5949.

Yang, Q., D. Co, J. Sommercorn, and N.K. Tonks. 1993. Cloning and expression of PTP-PEST: A novel, human, nontransmembrane protein tyrosine phosphatase. J. Biol. Chem. (in press).

Yang, X., K.T. Seow, S.M. Bahri, S.H. Oon, and W. Chia. 1991. Two Drosophila receptor-like tyrosine phosphatase genes are expressed in a subset of developing axons and pioneer neurons in the embryonic CNS. Cell 67:661.

Yu, Q., T. Lenardo, and R.A.Weinberg. 1992. The N-terminal and $\mathrm{C}$-terminal domains of a receptor tyrosine phosphatase are associated by non-covalent linkage. Oncogene 7: 1051.

Zheng, X.M., Y. Wang, and C.J. Pallen. 1992. Cell transformation and activation of pp $60^{\text {c-src }}$ by overexpression of a protein tyrosine phosphatase. Nature 359: 336. 